\title{
Collapse of Bell's Theorem
}

\author{
Guangye Chen \\ Shanghai Jiao Tong University, Shanghai, China \\ Email: gychen@sjtu.edu.cn
}

How to cite this paper: Chen, G.Y. (2019) Collapse of Bell's Theorem. Journal of Modern Physics, 10, 1157-1165.

https://doi.org/10.4236/jmp.2019.1010076

Received: July 30, 2019

Accepted: August 25, 2019

Published: August 28, 2019

Copyright $\odot 2019$ by author(s) and Scientific Research Publishing Inc. This work is licensed under the Creative Commons Attribution International License (CC BY 4.0).

http://creativecommons.org/licenses/by/4.0/

\begin{abstract}
Bell's theorem founded on Bell's inequalities asserts that no local realistic theories can reproduce all quantum mechanical predictions for spin correlation of particle pairs. It is pointed out that the assumption of setting-independent probability makes Bell's inequalities not impose constraint on all local realistic models and thus constitutes a theoretical loophole of Bell's theorem. A counterexample is presented by showing that a local realistic model with appropriate probability density reproduces all quantum mechanical predictions. It becomes clear that experiments violate Bell's inequalities because the real correlation functions are not constrained by these inequalities. It is also exposed that, rigorous logical reasoning of counter factual deduction does not permit to exclude any premises of Bell's inequalities by a certain amount of experimental violations of these inequalities.
\end{abstract}

\section{Keywords}

Bell's Theorem, Bell's Inequalities, Local Realism, EPRB Experiment

\section{Introduction}

Bell's theorem has influenced some physics researches over a half of century. As the foundation of the theorem, Bell's original inequality [1] has inspired two variants $\mathrm{CHSH}$ (Clauser-Horne-Shimony-Holt) inequality and $\mathrm{CH}$ (Clauser-Horne) inequality for convenience of experimental implementation [2] [3]. Several authors also claimed proofs of the theorem without using inequality. These proofs include the GHZ (Greenberger-Horne-Zeilinger) paradox [4] and Hardy's paradox [5]. As all proofs without inequality commence with the quantum mechanical concept of entangled state, they involve inherent inconsistency in logic for experimental test to discriminate between quantum mechanical concepts and classical concepts. Therefore, we are confined to consider merely the original Bell's theorem founded on Bell's inequalities, which are derived totally from 
classical concepts and have been used as criterions in most experimental tests.

Since the publication of Bell's seminal work, about twenty Bell experiments have been performed [6]. Majority of these experiments yielded outcomes violating one of Bell's inequalities and meantime agreeing with quantum mechanical predictions. During the earlier period, this experimental fact had been interpreted as the evidence of completeness of quantum mechanics or as a proof that no hidden variable theories exist, as then it had been often claimed that quantum mechanics overwhelms local realistic theories experimentally. Indeed, if quantum mechanics were proved a complete theory, not merely correct in predicting some properties of quantum systems, all weird properties arisen from its interpretation would be accounted as real physical phenomena. But proof of completeness of quantum mechanics seems far more difficult than the contrary. It was recognized many years later that violation of Bell's inequalities and proof of completeness of quantum mechanics are two irrelevant things. The objective of all Bell experiments was turned to exclude local realistic theories. Yet there has been no conclusive answer up to now. For the advocates of Bell's theory, the pending situation is caused by remaining loopholes for experimental tests of Bell's inequalities, despite the fact that the two main recognized loopholes were closed simultaneously in one experiment [7]. For other physicists, however, Bell's theorem is dubious due to the basic concepts or skeptical assumption underlying Bell's inequalities.

Negation of Bell's theorem is from computer simulations and theoretical analyses. Only theoretical aspect is exemplified here. Tipler showed that quantum non-locality does not exist if observed quantum particles and observer are both assumed to obey quantum mechanics as in the many-world interpretation [8]; Hess and Philipp demonstrated that Bell's theorem will be invalid if Bell's inequalities cover an extended parameter space that includes instrument parameters correlated by both time and setting dependencies [9] [10]. Although above viewpoints or alike have aroused argument or strong opposition from Bell's followers, they represent right stance against abandoning local realism, which is the fundamental believing in almost all other established scientific theories.

So far, the fact of experimental violations of Bell's inequalities has been established. This fact explains that Bell's inequalities are not applicable to the pertinent experiments. In other words, they are invalid in physics. Because their mathematical derivations are too simple to be suspected, it is deduced that at least one of their premises is wrong. [Here, we see that the verification of Bell's theorem is somewhat logically strange in comparison with that of other rules or theorems in physics. The involved problem will be discussed in the Section 3]. Some physicists think the attribute of local realism associated with the expression of the spin correlation function must be ruled out. However, the assumption of setting-independent probability can also be responsible for the situation. This point has long been ignored but is fatal to Bell's theorem. Without any further analysis, we immediately identify this assumption to be a theoretical loophole of the theorem, because it is not a necessary condition for locality. A set- 
ting-dependent probability can also be factorized to guarantee the locality of a correlation function. Therefore, we are sure that Bell's inequalities do not impose constraint on all local models and for this reason their experimental violation cannot rule out locality theoretically.

Zhao showed the necessity of setting dependence of the probability [11]. While the assumption of setting-independent probability is indispensible for derivation of Bell's inequalities. Bell had indeed sensed that setting-independent probability might be one of the four possibilities invalidating his theorem [12]. He thus proposed a measure for compensation while the inequality was kept in use [13]. Regarding this issue, Clauser also proposed a countermeasure [2]. To evaluate the effect of these compensations is very difficult, but it is unlikely that they could make the Bell's inequality cover up all local models.

To avoid the mentioned problem, actually, one can circumvent Bell's inequalities by investigating a correlation function directly. That is, one can exploit a correlation function that obeys the general condition of locality and then examine its compatibility with experimental outcomes as well as quantum mechanical prediction. If the concerned properties of a correlation function can be worked out directly, why do we make a detour by investigating them through a fabricated constraint on it?

It has been demonstrated that quantum mechanical correlation could be reproduced by local models. To author's knowledge, Barut et al. advanced an instance with a simple model of a classical break-up process [14], and Gisin et al. with a local hidden variable model exploiting detection loophole [15]. As these demonstrations are not directly related to the correlation function in Bell's original proof, and do not reveal the flaw of Bell's inequalities, they do not disprove Bell's theorem.

In this paper, a counterexample to Bell's theorem is presented by demonstrating that a spin correlation function adopted from an extension of Bell's origin reproduces all quantum mechanical predictions under appropriate probability densities. It is explained that verification of Bell's theorem through certain amount of experimental violations of Bell's inequalities is logically prohibited even if these inequalities imposed constraint on all local models. In summary, the counterexample disproves Bell's theorem firmly, and the cause for which is that Bell's inequalities do not impose constraint particularly on real correlation functions.

\section{The Counterexample}

To avoid ambiguity, let us begin by making clear the precise scope of local realistic models that is referred in Bell's theorem. As generally understood and also explained by Greenberg et al. [16], "local realistic theories" means that there are no other restrictions except locality and reality on the probability density, the hidden variable and the measuring outcomes in the correlation expression in Bell's original proof. For conciseness, spin $1 / 2$ of particle is tacitly assumed in 
following discussion.

It is known that quantum mechanical prediction for the spin correlation of particle pairs in EPRB experiment is given by

$$
\left\langle\Psi\left|\sigma_{\boldsymbol{a}} \otimes \sigma_{\boldsymbol{b}}\right| \Psi\right\rangle=-\boldsymbol{a} \cdot \boldsymbol{b}
$$

where $\langle\Psi|=(|+\rangle \otimes|-\rangle-|-\rangle \otimes|+\rangle) \sqrt{1 / 2}$ is the singlet spin state for the two particles, and $\sigma_{a}=\boldsymbol{\sigma} \cdot \boldsymbol{a}$ and $\sigma_{\boldsymbol{b}}=\boldsymbol{\sigma} \cdot \boldsymbol{b}$ are the components of Pauli spin operator $\sigma$ in the directions of $\boldsymbol{a}$ and $\boldsymbol{b}$ respectively; $\otimes$ denotes the Kronecker product, $\boldsymbol{a}$ is the unit vector of the spin analyzer setting for particle 1 and $\boldsymbol{b}$ for that of particle 2; the single particle vectors $|+\rangle$ and $|-\rangle$ denote "spin up" and "spin down" with respect to some coordinate system.

On the other hand, Bell gave the correlation function of local realistic theories as [1]

$$
\mathrm{P}(\boldsymbol{a}, \boldsymbol{b})=\int \rho(\lambda) \mathrm{A}(\boldsymbol{a}, \lambda) \mathrm{B}(\boldsymbol{b}, \lambda) \mathrm{d} \lambda
$$

From the expression above Bell derived his inequality and found that quantum mechanical prediction does not obey it. This led to his famous theorem. It is easily found that the setting-independent probability in Equation (2) is indispensible for the derivation of Bell's inequality. As aforementioned, this premise limits the range of local models imposed by the inequality. In other words, that correlation of Equation (2) cannot reproduce the quantum mechanical prediction does not prevent the correlation given by other local models from reproducing all quantum mechanical predictions. Hence, we will extend the correlation of Equation (2) to a general form by considering a setting-dependent probability. Although the general correlation prohibits derivation of any Bell-type inequality, it can be used to solve the key concern whether local realistic model can yield prediction in agreement with experimental result as well as quantum mechanical prediction.

Before the formal proof, all terms in Equation (2) are explained as follows. $\mathrm{A}(\boldsymbol{a}, \lambda)$ and $\mathrm{B}(\boldsymbol{b}, \lambda)$ are two measuring outcomes for the spins of particle pairs respectively, which take the values either +1 or -1 . $\mathrm{P}(\boldsymbol{a}, \boldsymbol{b})$ is the spin correlation that is given as an expectation of the product $\mathrm{A}(\boldsymbol{a}, \lambda) \mathrm{B}(\boldsymbol{b}, \lambda)$ with the probability density $\rho(\lambda)$, which is commonly called the joint probability density. $\mathrm{P}(\boldsymbol{a}, \boldsymbol{b})$ has been also termed as joint detection probability in many literatures, because the measuring outcomes have not dimension. $\lambda$ is a hidden parameter introduced by Bell, ordinarily considered to be a vector with its terminal points uniformly distributed on the surface of a unit sphere.

It is known, the general condition of locality is expressed by the factorability of the probability [17]. So, when probability density depends on the settings, the general correlation is given by

$$
\mathrm{P}(\boldsymbol{a}, \boldsymbol{b})=\int_{\Lambda} \rho_{\boldsymbol{a}}(\boldsymbol{a}, \lambda) \rho_{\boldsymbol{b}}(\boldsymbol{b}, \lambda) \mathrm{A}(\boldsymbol{a}, \lambda) \mathrm{B}(\boldsymbol{b}, \lambda) \mathrm{d} \lambda
$$

where $\Lambda$ is the sample space of $\lambda, \rho_{a}(a, \lambda)$ is the probability density for measuring $\mathrm{A}(\boldsymbol{a}, \lambda)$, and $\rho_{\boldsymbol{b}}(\boldsymbol{b}, \lambda)$ for $\mathrm{B}(\boldsymbol{b}, \lambda)$. It is evident that Bell's origi- 
nal correlation is a special case of the correlation given by Equation (3). Besides its generality in locality, this general correlation highlights that the measurements are performed on two spatially-separate stations, while the correlation $\mathrm{P}(\boldsymbol{a}, \boldsymbol{b})$ is merely a result of experimental data processing. Particularly, the measuring data at each station is allowed processing alone, so the statistical quantities of $\mathrm{A}(\boldsymbol{a}, \lambda)$ and $\mathrm{B}(\boldsymbol{b}, \lambda)$ can be calculated independently. For instance, the expectation of $\mathrm{A}(\boldsymbol{a}, \lambda)$ is given as

$$
\mathrm{E}(\mathrm{A})=\int_{\Lambda} \rho_{\boldsymbol{a}}(\boldsymbol{a}, \boldsymbol{\lambda}) \mathrm{A}(\boldsymbol{a}, \lambda) \mathrm{d} \lambda
$$

By the considerations above, the two probability densities must satisfy

$$
\int_{\Lambda} \rho_{a}(\boldsymbol{a}, \lambda) \mathrm{d} \lambda=1 \text { and } \int_{\Lambda} \rho_{\boldsymbol{b}}(\boldsymbol{b}, \boldsymbol{\lambda}) \mathrm{d} \lambda=1
$$

respectively.

The ideal counter-correlation of particle pairs is also assumed

$$
\mathrm{A}(\boldsymbol{a}, \lambda) \mathrm{B}(\boldsymbol{a}, \lambda)=-1
$$

Following Bell's treatment, measuring outcomes can be designated

$$
\mathrm{A}(\boldsymbol{a}, \boldsymbol{\lambda})=\operatorname{sign}(\boldsymbol{a} \cdot \boldsymbol{\lambda}) \text { and } \mathrm{B}(\boldsymbol{b}, \boldsymbol{\lambda})=-\operatorname{sign}(\boldsymbol{b} \cdot \boldsymbol{\lambda})
$$

where sign(.) is the symbolic function.

Suppose that $\rho_{a}(\boldsymbol{a}, \lambda)=\kappa_{a}|\boldsymbol{a} \cdot \lambda|$ and $\rho_{\boldsymbol{b}}(\boldsymbol{b}, \lambda)=\kappa_{b}|\boldsymbol{b} \cdot \lambda|$ respectively, where $\kappa_{a}$ and $\kappa_{b}$ are normalizing coefficients respective to each probability density. With these designations and assumptions, Equation (3) becomes simply

$$
\mathrm{P}(\boldsymbol{a}, \boldsymbol{b})=-\kappa \int_{\Lambda}(\boldsymbol{a} \cdot \boldsymbol{\lambda})(\boldsymbol{b} \cdot \lambda) \mathrm{d} \lambda
$$

in which $\kappa=\kappa_{a} \kappa_{b}$, being not a normalizing coefficient for the so called joint probability (Appendix 1).

Then suppose that $\lambda$ is a random vector with its terminal points distributing uniformly on the surface of a sphere with radius $r=1 / 3 \pi$ (Appendix 2), and denote it as $\lambda=r(\cos \varphi \sin \theta, \sin \varphi \sin \theta, \cos \theta)$, where $\varphi$ is the azimuthal angle and $\theta$ is the polar angle of the spherical coordinates, we have by inserting it in Equation (8)

$$
\mathrm{P}(\boldsymbol{a}, \boldsymbol{b})=-\kappa\left(I_{1}+I_{2}+I_{3}+I_{4}\right)
$$

in which

$$
\begin{gathered}
I_{1}=r^{3} \int_{0}^{2 \pi} \int_{0}^{\pi}\left(a_{1} b_{1} \cos ^{2} \varphi \sin ^{2} \theta+a_{2} b_{2} \sin ^{2} \varphi \sin ^{2} \theta+a_{3} b_{3} \cos ^{2} \theta\right) \sin \theta \mathrm{d} \varphi \mathrm{d} \theta, \\
I_{2}=r^{3} \int_{0}^{2 \pi} \int_{0}^{\pi}\left(a_{1} b_{2}+a_{2} b_{1}\right) \sin \varphi \cos \varphi \sin ^{3} \theta \mathrm{d} \varphi \mathrm{d} \theta, \\
I_{3}=r^{3} \int_{0}^{2 \pi} \int_{0}^{\pi}\left(a_{1} b_{3}+a_{3} b_{1}\right) \cos \varphi \sin ^{2} \theta \cos \theta \mathrm{d} \varphi \mathrm{d} \theta
\end{gathered}
$$

and

$$
I_{4}=r^{3} \int_{0}^{2 \pi} \int_{0}^{\pi}\left(a_{2} b_{3}+a_{3} b_{2}\right) \sin \varphi \sin ^{2} \theta \cos \theta \mathrm{d} \varphi \mathrm{d} \theta,
$$

where $a_{i}$ and $b_{i}(i=1,2,3)$ are components of $\boldsymbol{a}$ and $\boldsymbol{b}$, respectively.

Simple calculations yield $I_{1}=(4 \pi / 3) r^{3} \boldsymbol{a} \cdot \boldsymbol{b}$ and $I_{2}=I_{3}=I_{4}=0$, and also 
$\kappa_{a}=\kappa_{b}=1 /\left(2 \pi r^{2}\right)$. Substituting these and $r=1 / 3 \pi$ into Equation (9), we finally obtain

$$
\mathrm{P}(\boldsymbol{a}, \boldsymbol{b})=-\kappa_{a} \kappa_{b} I_{1}=-\boldsymbol{a} \cdot \boldsymbol{b}
$$

which is exactly the right side of Equation (1), what quantum mechanics predicts.

Quantum mechanical predictions may also include that the expectations of particle spin at each terminal are equal to zero, that is,

$$
\mathrm{E}(\mathrm{A})=\mathrm{E}(\mathrm{B})=\mathbf{0}
$$

These are readily confirmed. For example, we have

$$
\mathrm{E}(\mathrm{A})=\int \rho_{\boldsymbol{a}}(\boldsymbol{a}, \boldsymbol{\lambda}) \mathrm{A}(\boldsymbol{a}, \boldsymbol{\lambda}) \mathrm{d} \lambda=\kappa_{a} \int|\boldsymbol{a} \cdot \lambda| \operatorname{sign}(\boldsymbol{a} \cdot \boldsymbol{\lambda}) \mathrm{d} \boldsymbol{\lambda}=\kappa_{a} \int(\boldsymbol{a} \cdot \boldsymbol{\lambda}) \mathrm{d} \lambda
$$

and then substitution of $\lambda$ as supposed previously and a calculation gives $\mathrm{E}(\mathrm{A})=0$.

Up to this point, we have presented the counterexample to Bell's theorem. Definitely, the correlation function that we have achieved, just like quantum mechanical prediction, is not constrained by Bell's inequalities. This is the true reason for experimental violation of these inequalities.

\section{Logical Issue with Experimental Verification of Bell's Theorem}

Let us scrutinize a logical issue with application of Bell's inequalities. We have made clear that Bell's inequalities only impose constraint on partial local models. Moreover, even if this flaw does not exist, there is also a logical obstacle with application of these inequalities. To exclude the premises of these inequalities by experimental violations belongs to the counter factual deduction of logical reasoning. However, to be rigorous, one will find in this logical deduction that a certain amount of experiments cannot substitute a theoretical proposition, because a theoretical proposition might involve indefinite or even infinite amount of experiments. Hence, to exclude the premises of Bell's inequalities by experimental violations of them is not permitted by rigorous logical reasoning.

\section{Discussion of Bell's Model}

In his seminal paper, Bell intended to implement the guessed EPR's idea of hidden variable model. As he stated, “...that idea will be formulated mathematically and shown to be incompatible with the statistical predictions of quantum mechanics." But his inequality and its variants are actually unrelated to hidden variable theory, because these inequalities are derived totally from classical concepts. The introduced parameter $\lambda$ has nothing to do with completing the description of quantum mechanics though it has been termed hidden variable or parameter. The word of local hidden theories or models has concealed the inappropriateness of the assumption of setting-independent probability. Besides limiting the coverage of Bell's inequalities, actually, the setting-independent proba- 
bility causes incompatibility with certain experimental observation. If the second moment of the outcome on one terminal is observed in the EPRB-type experiment with the photons of linear polarization, it will make the established fact like Malus' rule unpredictable. The demonstration is omitted here as it is very simple. Therefore, the assumption of setting-independent probability is inconsistent with what occurs in actual measuring process. Actually, that CHSH inequality was derived without any consideration of measuring process [18] has already hinted such inequality not for experimental test but to be a trivial mathematical relation.

\section{Conclusions}

As a necessary assumption of Bell's inequalities, the setting-independent probability makes these inequalities not impose constraint on all correlation functions representing local realistic models, and is also apparently false for actual experimental measurement. Existence of the counterexample further manifests that Bell's inequalities particularly do not impose constraint on real correlation functions. The revealed fatal flaw of these inequalities explains why they have been violated by experiments. Moreover, even if these inequalities had not the flaw, they would be useless for experiment because certain amount of experiments cannot substitute a theoretical proposition in counter factual deduction. It is concluded that, Bell's theorem is false because Bell's inequalities are trivial mathematical relations that, due to an unsuitable assumption of probability, lack essential connection with the real measuring process of the pertinent experiments.

Meanwhile, a point of view should be stated. Collapse of Bell's theorem does not deny the conflicts between classical concepts and some weird notions by the orthodox interpretation of quantum mechanics. And what Einstein had insisted that these conflicts are caused by incompleteness of quantum mechanics in description of microscopic systems appears rather reasonable.

\section{Acknowledgements}

The author wish to thank professor Karl Hess of the University of Illinois for a helpful proposal.

\section{Conflicts of Interest}

The author declares no conflicts of interest regarding the publication of this paper.

\section{References}

[1] Bell, J.S. (1964) Physics Physique Fizika, 1, 195-200. https://doi.org/10.1103/PhysicsPhysiqueFizika.1.195

[2] Clauser, J.F., Horne, M.A., Shimony, A. and Holt, R.A. (1969) Physical Review Letters, 23, 880-884. https://doi.org/10.1103/PhysRevLett.23.880 
[3] Clauser, J.F. and Horne, M.A. (1974) Physical Review D, 10, 526-535. https://doi.org/10.1103/PhysRevD.10.526

[4] Greenberger, D.M., Horne, M.A. and Zeilinger, A. (1989) Going beyond Bell's Theorem. In: Kafatos, M., Ed., Bell s Theorem, Quantum Theory, and Conceptions of the Universe, Kluwer Academic, Dordrecht, The Netherlands, 69-72.

https://doi.org/10.1007/978-94-017-0849-4_10

[5] Hardy, L. (1993) Physical Review Letters, 71, 1665-1668. https://doi.org/10.1103/PhysRevLett.71.1665

[6] Clauser, J.F. (2017) Bell's Theorem, Bell Inequalities, and the "Probability Normalization Loophole". In: Bertlmann, R. and Zeilinger, A., Eds., Quantum [UN] Speakables II. The Frontiers Collection. Springer, Cham, 451-484. https://doi.org/10.1007/978-3-319-38987-5_28

[7] Hensen, B., et al. (2015) Nature, 526, 682-686. https://doi.org/10.1038/nature15759

[8] Tipler, F.J. (2014) Proceedings of the National Academy of Sciences of the United States of America, 111, 11281-11286. https://doi.org/10.1073/pnas.1324238111

[9] Hess, K. and Philipp, W. (2001) Proceedings of the National Academy of Sciences of the United States of America, 98, 14224-14227. https://doi.org/10.1073/pnas.251524998

[10] Hess, K. and Philipp, W. (2004) Proceedings of the National Academy of Sciences of the United States of America, 101, 1799-1805.

https://doi.org/10.1073/pnas.0307479100

[11] Zhao, H.L. (2013) Journal of Experimental and Theoretical Physics, 117, 999-1010. https://doi.org/10.1134/S1063776113140070

[12] Bell, J.S. (1987) Speakable and Unspeakable in Quantum Mechanics. Cambridge, New York, 154.

[13] Bell, J.S. (1971) Introduction to the Hidden-Variable Question. In: d'Espagnat, B., Ed., Foundations of Quantum Mechanics, Academic, New York, 171-181.

[14] Barut, A.O. and Meystre, P. (1984) Physics Letters, 105, 458-462.

[15] Gisin, N. and Gisin, B. (1999) Physics Letters A, 260, 323-327. https://doi.org/10.1016/S0375-9601(99)00519-8

[16] Greenberg, D.M., Horne, M.A., Shimony, A. and Zeilinger, A. (1990) American Journal of Physics, 58, 1131-1143. https://doi.org/10.1119/1.16243

[17] Clauser, J.F. and Shimony, A. (1978) Reports on Progress in Physics, 41, 1881-1927. https://doi.org/10.1088/0034-4885/41/12/002

[18] Hess, K. and Philipp, W. (2001) Proceedings of the National Academy of Sciences of the United States of America, 98, 14228-14233.

https://doi.org/10.1073/pnas.251525098 


\section{Appendix 1}

Due to the two constraints Equations (5a) and (5b), the product

$\rho_{a}(\boldsymbol{a}, \lambda) \rho_{\boldsymbol{b}}(\boldsymbol{b}, \lambda)$ generally termed as the joint probability is actually not a normalized probability density. The proof is given as follows. The product can be conceived as a degenerated joint probability from $\rho_{\boldsymbol{a}}(\boldsymbol{a}, \lambda) \rho_{\boldsymbol{b}}(\boldsymbol{b}, \gamma)$ for two random variables $\lambda$ and $\gamma$, when $\gamma$ goes to equate $\lambda$ and both have the same sample space $\Lambda$. With this consideration, we have

$$
1=\int_{\Lambda} \int_{\Lambda} \rho_{\boldsymbol{a}}(\boldsymbol{a}, \lambda) \rho_{\boldsymbol{b}}(\boldsymbol{b}, \gamma) \mathrm{d} \lambda \mathrm{d} \gamma>\int_{\Lambda} \int_{\Lambda} \rho_{\boldsymbol{a}}(\boldsymbol{a}, \lambda) \rho_{\boldsymbol{b}}(\boldsymbol{b}, \gamma) \delta(\lambda-\gamma) \mathrm{d} \lambda \mathrm{d} \gamma
$$

where $\delta(\cdot)$ is Dirac function. The most right part of the above is exactly $\int_{\Lambda} \rho_{\boldsymbol{a}}(\boldsymbol{a}, \lambda) \rho_{\boldsymbol{b}}(\boldsymbol{b}, \lambda) \mathrm{d} \lambda$.

It is noted that all Bell inequalities were derived by that the joint probability fulfills the normalization condition. This normalization would be justified if the joint probability were for ordinary two random events. But the two random outcomes in the considered experiment are dependent on one parameter $\lambda$ with the expectation of their product being not determined by a normalized joint probability, as demonstrated above. This exceptional property was not noticed in derivations of Bell's inequalities.

\section{Appendix 2}

We deliberately choose $r=1 / 3 \pi$ rather than ordinarily $r=1$ to obtain a normalized correlation function in end, though this operation is essentially unnecessary. This means that a normalization of the correlation function is carried out in advance. Otherwise, it is readily found that the final correlation function will be $\mathrm{P}(\boldsymbol{a}, \boldsymbol{b})=-(1 / 3 \pi) \boldsymbol{a} \cdot \boldsymbol{b}$. In a view of geometry, a series of data represents a line, and the essence of a correlation coefficient represents the cosine of the angle made by two lines. The value of the cosine is independent of either length of the two lines. While a correlation function is the extension of the correlation coefficient when it depends on a variable. In practice, it is well known to an experimental physicist or an engineer of signal processing that a scaling of experimental data has to be carried out in order to verify a theoretical correlation function. This scaling is really equal to the normalization of a correlation function, entailing that the absolute magnitude of a correlation function makes nonsense or that correlation functions only different in magnitude are equivalent. Please excuse the author for the wordy explanations above, since some theorists might ignore this tiny issue. For instance, Bell did not treat $-(1 / 3) \boldsymbol{a} \cdot \boldsymbol{b}$ as equivalent to $-\boldsymbol{a} \cdot \boldsymbol{b}$ in his seminal paper [1]. 